\title{
Surgical correction of senile entropion of the lower lid with fascia lata sling
}

\author{
S. N. M. RIZK AND A. K. MISHRA
}

From the Nottingham Eye Hospital

SUMMARY A method of surgical correction of senile entropion of the lower lid with heterogenous fascia lata sling is described with no tissue loss and minimum of surgical trauma.

Numerous surgical techniques described in the past for correction of senile entropion of the lower lid indicate that there is no reliable single method to deal with the problem. The object of this paper is to describe a surgical technique using a heterogenous fascia lata sling for the correction of senile entropion.

\section{Surgical pathology}

Jones (1960) has described the following changes in the senile eye: $(a)$ The senile absorption of orbital fat causes some degree of enophthalmos, which

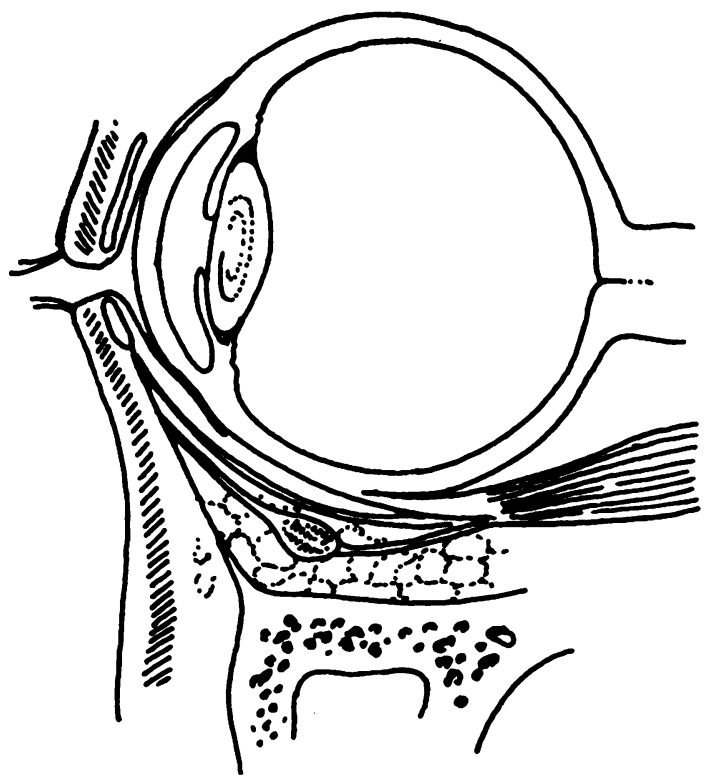

Fig. 1 Cross-section of lower lid of a normal adult

Address for reprints: Mr S. N. M. Rizk, FRCS, Nottingham Eye Hospital, The Ropewalk, Nottingham

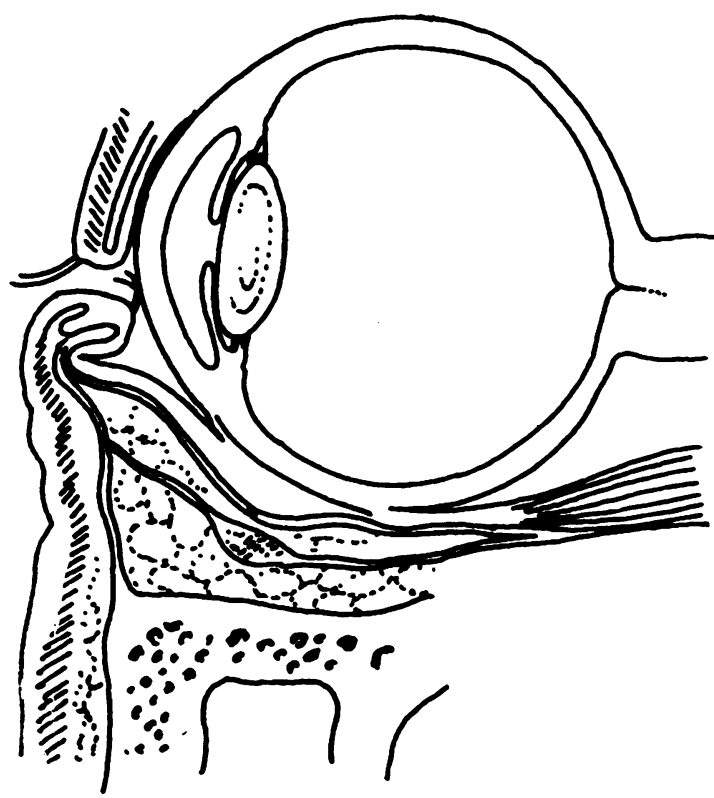

Fig. 2 Cross-section of lower lid in senile entropion

reduces the pressure of the globe against tarsi. (b) The skin and superficial fascia become atonic, redundant, and less adherent to underlying preseptal muscle. (c) The lower preseptal muscle becomes less fixed to the underlying septum orbitale and loses its pressure against the base of the tarsus and moves upwards over the pretarsal during contractions of the muscle (Fig. 1). (d) The deep fascial layers attached to the base of the tarsus become relaxed not only by the enophthalmos but also by the usual atony accompanying the ageing process. These factors reduce the pressure against the lower border of the tarsus and increase it against the upper border, allowing the tarsus to turn in at a right-angle (Fig. 2). 


\section{Operative technique}

The skin of the lower lid is cleaned with $1 \%$ chlorhexidine solution. It is then infiltrated with about $2 \mathrm{ml}$ of $1 \%$ lignocaine-adrenaline $\left(\begin{array}{ll}1 & 100000\end{array}\right)$ along its entire length from the medial canthus to just outside the lateral orbital margin. The injection is given subcutaneously.

Two vertical incisions about $5 \mathrm{~mm}$ long are made, one over the medial canthal tendon about 3 to $4 \mathrm{~mm}$ from the medial canthus and the other over the outer orbital rim level with the outer canthus. The subcutaneous tissue is dissected with sharp-

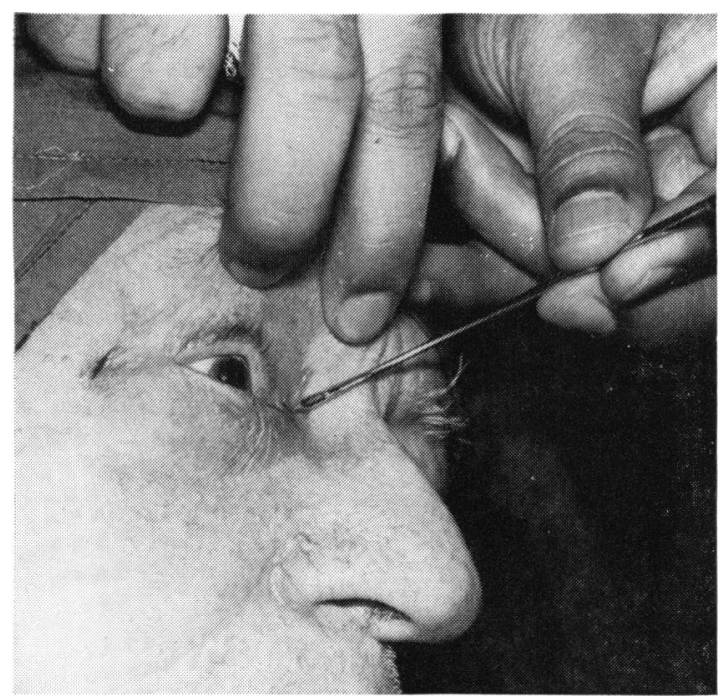

Fig. 3 The aneurysm needle in the lower lid

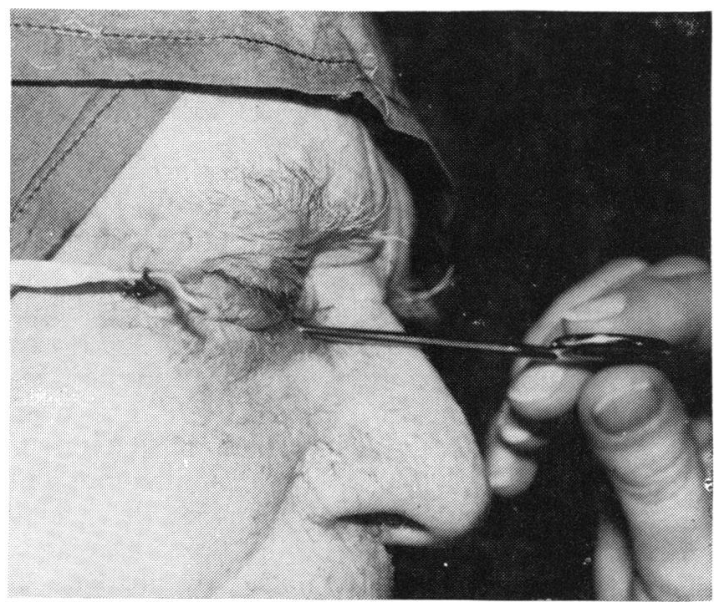

Fig. 4 The fascia lata is being threaded into the eye of the aneurysm needle

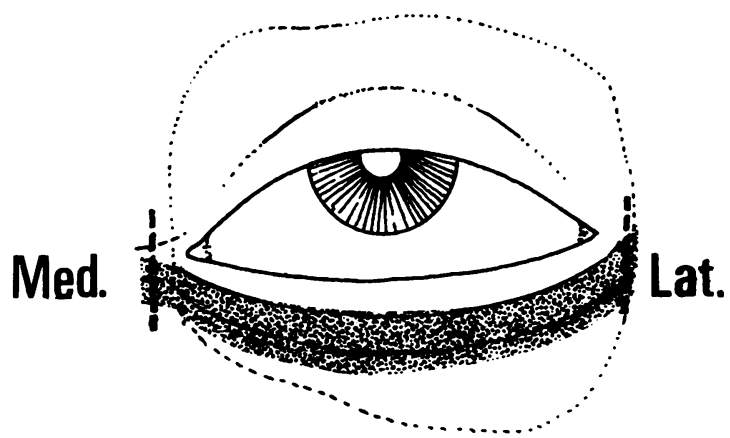

Fig. 5 The eye with fascia lata sling in position

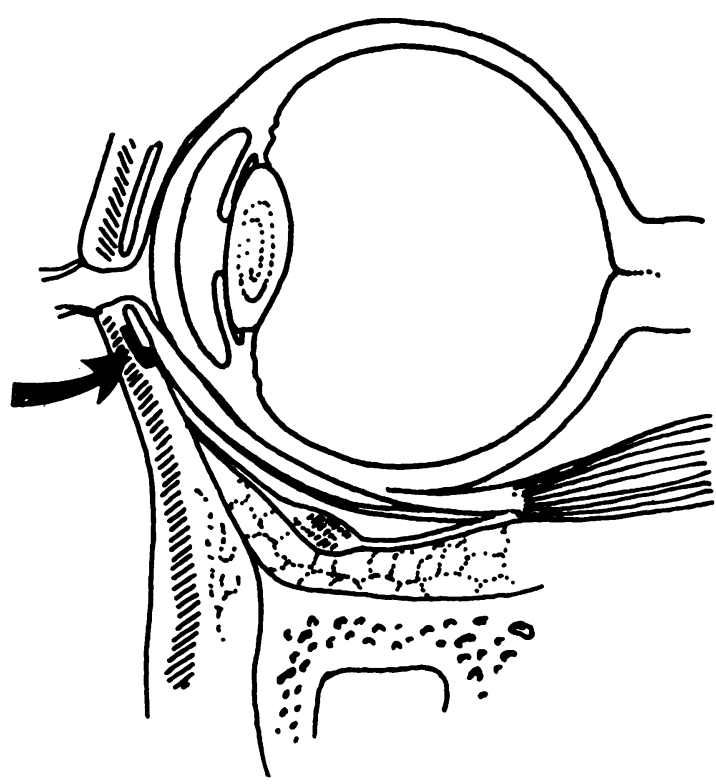

Fig. 6 Cross-section of the eye with fascia lata sling in position

pointed scissors to expose the medial canthal tendon, and the same procedure is carried out at the outer orbital rim to expose the periosteum. A sharp-pointed aneurysm needle is introduced through the lateral incision and directed medially level with the lower half of the lower tarsal plate, its tip being constantly felt with the fingers until its eye comes out through the medial incision. If the bridge of the nose is not very prominent it is sometimes easier to pass the needle from the medial to the lateral side (Fig. 3).

A strip of heterogenous fascia lata (derived from oxen) cut about $3 \mathrm{~mm}$ wide and $10 \mathrm{~cm}$ long and washed in saline solution is threaded through the aneurysm needle, which is withdrawn, taking the strip with it (Fig. 4). One end of the strip is stitched 
to the medial canthal tendon with 5-0 Dexon sutures. The strip is now made slightly taut, the right tension being judged by the appearance of a depression in the skin overlying the strip of fascia lata. The lateral end of the strip is now firmly stitched to the periosteum covering the outer orbital rim. The excess of strip is cut off and buried under the orbicularis. The wounds are then sutured in layers.

\section{Results}

This surgical procedure has so far been carried out on 62 cases. One phthisical eye is included in the series. The follow-up period varied from 6 weeks to 2 years. Apart from 2 cases, in which the entropion recurred 6 weeks after surgery, the results were very encouraging. The same procedure was repeated on the 2 patients with recurrence, with a satisfactory result. Most of the cases have been followed up for over 2 years now without any recurrences. None of these patients showed any obvious scar or cosmetic problem. None had any infection or postoperative rejection.

\section{Discussion}

In this technique there is no tissue loss and very little surgical trauma. The fascia lata strap supports the lower part of the tarsal plate (Fig. 5) and orbital septum and holds it near the globe (Fig. 6). Transfixing the orbicularis fibres by tunnelling the strip through them may enhance the effectiveness of the operation by preventing the muscle from advancing upward on the tarsal plate. Injury to important structures is easily avoided, and there is good haemostasis. Use of Dexon sutures avoids the need to remove them at a later date.

A similar procedure has been described by Ray A. Elliott, Jr.(1962), who used autogenous fascia lata. He reported 6 cases, and results were $100 \%$ satisfactory.

Fascia Lata is a sterile preparation marketed by Ethicon.

\section{References}

Elliott, R. A., Jnr. (1962). Correction of senile entropion with fascia lata graft. Plastic and Reconstructive Surgery, 29, 698-701.

Jones, L. T. (1960). The anatomy of the lower eyelid and its relation to the cause and cure of entropion. American Journal of Ophthalmology, 49, 29-36. 